\title{
La invención del hombre desde la exteriorización tecno-lógica: una relación asimétrica*
}

Fecha de entrega: 12 de febrero de 2018

Fecha de evaluación: 7 de junio de 2018

Fecha de aprobación: 30 de junio de 2018

\author{
Steve Macraigne*** \\ María Daniela Parra Bernal*** \\ Sergio Néstor Osorio García****
}

\section{Resumen}

Uno de los problemas centrales que el filósofo francés Bernard Stiegler ha abordado para sostener una constitución tecno- lógica de la humanidad, es la cuestión del origen del hombre. El asunto recae en

* Artículo de investigación producto del proyecto de investigación de alto impacto IMP HUM 2298: "Constitución Tecnológica de la Humanidad. Hacia una bioética fundamental. Segunda fase". Avalado por la Vicerrectoria de Investigación de la Universidad Militar Nueva Granada. Citar como: Macraigne, S., Parra, M.D. y Osorio, S.N. (2018). La invención del hombre desde la exteriorización tecno-lógica: una relación asimétrica. Cuadernos de Filosofía Latinoamericana, 39(119), 215-237. DOI: http://www. doi.org/10.15332/25005375.5057

* Asistente de investigación del proyecto de investigación de alto impacto IMP HUM 2298. Docenteinvestigador en la Facultad de Medicina de la Fundación Universitaria de Ciencias de la Salud. Miembro del grupo de investigación BioethicsGroup, línea Bioética global y complejidad, de la UMNG desde el año 2012. Bogotá, Colombia. Correo electrónico: stevemac53@yahoo.fr

*** Asistente graduada en el programa de doctorado en bioética de la Universidad Militar Nueva Granada, vinculada al proyecto de investigación de alto impacto IMP HUM 2298. Miembro del grupo de investigación BioethicsGroup, línea Bioética global y complejidad, de la UMNG desde el año 2017. Bogotá, Colombia. Correo electrónico: maradanielaparrabernal@gmail.com

**** Docente de tiempo completo de la Facultad de Educación y Humanidades de la Universidad Militar Nueva Granada. Investigador principal del proyecto de investigación de alto impacto IMP HUM 2298. Investigador Lider del grupo de investigación BioethicsGroup de la UMNG. Bogotá, Colombia. Correo electrónico: Sergio.osorio@unimilitar.edu.co 
preguntarse si es posible pensar quién es el hombre desde esta tesis, sin volver a la metafísica o la filosofía trascendental. Esta posición ha de estar enraizada en una ontología que aprehenda el componente causal a nivel relacional, dejando de lado las explicaciones en las cuales la causa es substancialista. Para dar desarrollo a esta propuesta, este artículo se compone de dos partes generales: la primera está destinada a presentar hasta dónde sigue y en dónde se separa Stiegler de la concepción zoo-antropológica de André Leroi-Gourhan sobre el origen del hombre; para luego explicar las bases epistemológicas y ontológicas sobre las que Stiegler erige sus tesis tecno-ontológica de la humanidad; a saber: los fundamentos de la transducción simondoniana y la diferancia derridiana.

Palabras clave: filosofía de la tecnología, bioética, ontología, Bernard Stiegler, Gilbert Simondon, Jaques Derrida, Ezequiel Di Paolo.

\section{The invention of man from the techno-logical externalization: an asymmetric relationship}

\section{Abstract}

One of the central problems that the French philosopher Bernard Stiegler has tackled to sustain a techno-logical constitution of humanity is the question of the origin of man. The question lies in asking whether it is possible to think the origin of the humankind from this thesis, without returning to metaphysics or transcendental philosophy. This position must be rooted in an ontology that apprehends the causal component at the relational level, leaving aside the explanations in which the cause is substantialist.

In order to develop this proposal, this article is composed of two general parts: the first one is destined to present up to where Stiegler follows and up to where he separates from the zoo-anthropological conception of André Leroi-Gourhan about the origin of man; to then explain the epistemological and ontological bases on which Stiegler erects his 
techno-ontological thesis of humanity; namely: the foundations of Simondonian transduction and Derridian différance.

The Stielgerian thesis of a common and relational origin of technique and man in a process of technical externalization, cannot be thought from a previous interiority. The difficulty is to understand how, from a relational ontology, we can account for the difference of man in life, his birth in continuity and differentiation with the animal. To clarify this relational dynamic and overcome the paradoxes that Stiegler's discourse seems to generate, we discussed the opportunity for a terminological convergence with the perspective of biological enactivity worked by $\mathrm{Di}$ Paolo. In this sense, we could speak of symmetry and relational asymmetry, of relational polarization leading to the establishment of poles or polarities and thus clarify the continuity (community) and the rupture (separation) that exists between life and man, between the technicality of the live and human technology.

Keywords: philosophy of technology, bioethics; ontology, Bernard Stiegler, Gilbert Simondon, Jaques Derrida, Ezequiel Di Paolo.

\section{Introducción}

Uno de los problemas centrales que Stiegler ha abordado para sostener una constitución tecno- lógica de la humanidad, es la cuestión del origen del hombre. Esta cuestión es vertebral de la tesis stiegleriana, a saber: no es posible pensar en un hombre que no sea técnico (o protético). Todo el asunto recae, entonces, en preguntarse si es posible pensar quién es el hombre desde esta tesis, sin recaer en la metafísica o la filosofía trascendental que ofrecen del hombre una visión antropomórfica que lo aísla dentro y del resto de la vida y del ser. Para salir de esta tendencia, Stiegler pregunta por la invención del hombre desde una perspectiva que se reivindica zoológica, siguiendo inicialmente a la vía abierta por Leroi-Gourhan. Tradicionalmente, este asunto sería abordado desde una perspectiva clásica para pensar en un inventor (Quién) y en algo que es inventado (Qué). Esto conduciría a buscar, por una parte, un creador fuera de lo creado, y, por otra, a considerar la explicación del 'origen' desde una causa previa. Tanto el creador como la causa se verían, desde esta perspectiva filosófica tradicional, exterior a -respectivamente- lo creado y al origen que explican. No obstante, hay que aclarar que Stiegler reivindica una comprensión 
del "origen del ser humano" que precisamente se sale de esta perspectiva causal, esencialista o substancialista.

En principio, la novedad del pensamiento de Stiegler es que busca dar cuenta de la invención (nacimiento u origen) del hombre sin apelar a un creador, una esencia o causa que, como origen exterior al hombre, contendría sin embargo toda la explicación de su ser. La técnica tiene para Stiegler no sólo un papel instrumental, sino también uno antropo-ontológico. Sin embargo, esta proposición ha de estar enraizada en una ontología que aprehende el componente causal a nivel relacional, es decir rechazando las explicaciones en las cuales la causa se concentra en o proviene de un solo término. Ver la causa o el origen en uno solo de los términos en relación, es regresar a una visión sustancialista (uni-causal) del ser humano en la cual éste es el inventor y la técnica lo inventado. En cambio, si se asume que la técnica y el hombre se co-constituyen, significa que podemos pensar, en principio, que la técnica es tan causa del hombre que éste causa de aquella.

La propuesta stiegleriana sobre la técnica, en relación con el proceso de invención del hombre, no busca superar a la metafísica, sino que se presenta como una forma no-metafísica de pensar la técnica y el hombre. En vez de pensar que el hombre es el inventor de la técnica, que es quién inventa al qué (como si existiera el hombre antes de la técnica) se va a proponer una hipótesis antropológica y filosófica distinta: que la técnica y el hombre son más bien co-originarios; o, de manera más provocativa, invirtiendo la interpretación clásica, que es la técnica quién inventa al hombre (que se vuelve el qué de la invención, el inventado, el medio).

Cuando Stiegler introduce esta tesis en el primer tomo de La técnica y el tiempo, se centra primero en las teorías de la evolución técnica que interpretan la evolución técnica como evolución de la técnica en el tiempo (Stiegler, 2003, primer capítulo de la primera parte). Luego, se detiene en mostrar que antropología y tecnología no son independientes, y que no se puede pensar el origen y la evolución del hombre fuera de la técnica (Stiegler, 2003, segundo capítulo de la primera parte). Así pues, la técnica no está sencillamente en el tiempo y la pregunta por la técnica es también la pregunta por el tiempo, por la temporalización de la vida del hombre. Como un tercer paso argumentativo, Stiegler desarrolla propiamente esta visión filosófica sobre la técnica y el hombre (Stiegler, 2003, tercer capítulo de la primera parte). Para ello, adopta una perspectiva paleo-antropológica (la de Leroi-Gourhan) y la discute filosóficamente 
a la luz de la diferancia (Derrida) y la individuación (Simondon). Apartándose de la explicación propuesta por Leroi-Gourhan, va a sostener que la técnica y el hombre son co-originarios desde la "primera" exteriorización técnica: el hombre se inventa en la técnica inventando la herramienta -exteriorizándose tecno-lógicamente (Stiegler, 2002, p. 213). La interpretación de este nacimiento del hombre, que va constituir el centro de la propuesta de Stiegler, no radica precisamente en uno de los términos en relación (la técnica o el hombre):

Ahora bien, el hombre es aquí el “interior": no hay exteriorización que no designe
un movimiento del interior hacia el exterior. Sin embargo, el interior es inven-
tado por ese movimiento: no puede, por lo tanto, precederlo. Por consiguiente,
interior y exterior se constituyen en un movimiento que inventa al uno)' al otro
a la vez: un movimiento en el que se inventan el uno en cl otro, como si hubiera
una mayéutica tecno-lógica de eso que se llama el hombre. (Stiegler, 2002, p. 213)

Podríamos sin duda hablar de origen transductivo (Simondon) para caracterizar la propuesta de Stiegler: se trata de un origen no sustancial, en el cual lo que está en juego se encuentra al nivel de la relación que une los términos más que en los términos en relación.

Para dar desarrollo a esta propuesta, el presente texto se compone de dos partes fundamentales: la primera está destinada a presentar hasta dónde sigue y en dónde se separa Stiegler de la concepción zoo-antropológica de André Leroi-Gourhan sobre el origen del hombre; para luego explicar las bases epistemológicas y ontológicas sobre las que Stiegler erige sus tesis tecno-ontológica de la humanidad. La segunda se destina a presentar problemas y reflexiones sobre la propuesta stiegleriana desde sus fundamentos en la transducción simondoniana y la diferancia derridiana. Para aclarar esta dinámica relacional y superar las paradojas que parece generar el discurso stiegleriano, discutimos la oportunidad de una convergencia terminológica con la perspectiva de la enactividad biológica trabajada por Ezequiel Di Paolo (2013).

\section{La constitución tecno-lógica de la humanidad}

\section{Noción evolutiva del origen del hombre}

Stiegler persigue un rumbo distinto para entender quién es el hombre, a saber: la ruta de la zoología evolutiva que le marca André Leroi- Gourhan. Sin embargo, la propuesta 
de Leroi-Gourhan no conviene del todo a la construcción de la tesis stiegleriana sobre la constitución tecno-lógica de la humanidad, pues cae en las mismas aporías a las que conducen las ideas clásicas sobre el “origen”.

Lo que encuentra Stiegler como un punto de partida es que, desde esa vía zoológica, la pregunta se dirige hacia el proceso de la invención del hombre desde la vida y la animalidad, más que a una explicación esencial o trascendental. De forma que la cuestión aparece aquí dentro de una perspectiva evolutiva en la que el hombre se concibe como una ramificación en el entramado de la vida. En este sentido, la idea de su origen estaría remitida a su invención dentro de un proceso de individuación vital. Así, la pregunta no sería quién es el creador o cuál es su causa, sino cómo tendríamos que entender la vida para concebir cómo aparece, dentro de ella, el hombre como una forma diferente de vida. La idea que va a defender Stiegler es que esta diferenciación del hombre del resto de lo vivo se da gracias a la técnica.

La tesis stiegleriana en esta parte es la siguiente: el proceso de hominización, la 'invención del hombre', empieza con la diferancia técnica como proceso de exteriorización de la memoria, experiencia o vivencia del individuo en objetos técnicos. A la vez y en conjunto, estos objetos conforman una memoria en exterioridad de las experiencias vividas (epi) a nivel de la especie (filo), un medio tecnológico (epifilogénético) que media la constitución del ser humano (o, desde Simondon, que conforma el medio pre-individual para la individuación psíquica y colectiva propiamente humana). Este proceso de exteriorización técnica es correlativo de uno de interiorización cognitiva (humana) y corresponde también a la doble determinación entre la técnica y el hombre.

Desde la perspectiva de Leroi-Gourhan, se puede hablar de un hombre como tal cuando el proceso de hominización se estabiliza en el Neantropus, momento en el que el desarrollo de las capacidades cerebrales culmina. Por su parte, Stiegler muestra que, en el proceso de hominización, la corticalización (aumento de la capacidad cerebral) se desarrolla al ritmo de la diversificación de las herramientas fabricadas e usadas por los hombres prehistóricos. Esta co-evolución genética-cognitiva es lenta y resulta ser una consecuencia de la bipedestación ("todo empieza por los pies") y de la liberación correspondiente de la mano, que permiten la exteriorización técnica y el desarrollo de la capacidad cerebral. 
Por tanto, ¿quién inventa a qué? Pareciera que es la técnica la que inventa al hombre, pues a nivel evolutivo (filogenético), su capacidad cerebral no aparece como la causa del surgimiento y la evolución técnica, sino como su consecuencia; consecuencia del proceso de exteriorización que cambia el medio de la individuación vital y de la selección natural (genética).

En el proceso de hominización, y usando la terminología paleo-antropológica que Stiegler retoma de Leroi-Gourhan, esta etapa corresponde al Zinjantropus. Sin embargo, con el Neantropus -el sucesor de Zinjantropus en el proceso- Leroi-Gourhan ve un "segundo origen" del hombre: mientras que la técnica sigue evolucionando y diversificándose de manera rápida y que surgen huellas de actividades humanas "nuevas" (ritos funerarios, tumbas, pinturas), el proceso biológico de desarrollo cerebral (corticalización) del hombre se ha estabilizado. La interpretación de este desacople (entre evolución biológica y técnica), su sentido para la antropología y la tecnología, es lo que alejará a Stiegler de Leroi-Gourhan. En este momento, Stiegler considera que Leroi-Goubhan repite el gesto de la tradición, viendo en este segundo origen (del Neantropus) el "verdadero" origen del hombre.

Para Stiegler, el hombre es Sapiens (tiene lenguaje simbólico, abstracto, anticipa la muerte y es sociable) a partir del momento en que se origina el proceso de exteriorización técnica, inclusive si es de forma rudimentaria. Es decir, la ruptura por la cual surge la humanidad como distinta, dentro de la vida, se da a partir de la exteriorización técnica y no está causada, como se suele pensar (y como lo seguirá pensando Leroi-Gourhan), por el desarrollo de la capacidad cerebral (culminación del proceso de corticalización).

De lo dicho, se desprende que la cuestión de la invención del hombre nos remite a una ambigüedad genitiva, pues no sabemos si es el hombre quien se inventó algo, la técnica, o si es el hombre el que está siendo inventado por ella. Para Stiegler, pareciera que es 'más' la técnica que inventa al hombre que lo contrario: pues a nivel evolutivo (filogenético), su capacidad cerebral no es la causa del surgimiento y de la evolución técnica, sino la consecuencia de ésta en tanto proceso de exteriorización de la memoria individual. Y este proceso de “invención” empieza con el Zinjantropus, no cuando el proceso del desarrollo cerebral del hombre se estabiliza. Esto último será lo que LeroiGourhan entenderá como un "segundo origen", como un salto hacia lo humano que tiene lugar con el Neantropus. 
Si las cosas se piensan así, llegaría un momento en que el proceso de constitución de las capacidades cognitivas del hombre se estabilizaría (memoria genética) mientras que la técnica seguiría evolucionando, provocando un desacoplamiento entre el medio biológico interior y el medio tecnológico exterior. El sentido que reviste esta ruptura será uno de los puntos de separación entre Stiegler y Leroi-Gourhan. Para el paleantropólogo, es allí donde tiene cabida lo propiamente humano, separado de la técnica, mientras que, para el filósofo, esta relación ha determinado la constitución de la humanidad desde que el hombre fue Zinjantropus.

Toda la discusión, a nivel filosófico, es saber si uno considera como origen el cambio transductivo inaugurado por la exteriorización técnica (correlativo de una interiorización cognitiva) o el resultado de este proceso, que culmina con el 'llenado cortical' (en el desarrollo de la capacidad cerebral). ¿Cuál es el origen “verdadero”? ¿El cambio transductivo en la individuación vital, la diferancia de una diferancia o el resultado de este proceso? ¿En función de qué tipo de origen decimos quién es el hombre? Para Stiegler, la ruptura que opera en continuidad con la vida es la ruptura técnica: "ruptura" porque se trata de un cambio de régimen transductivo por el cual se co-inventan la técnica y el hombre; y "continuidad" porque este cambio, al principio, "casí" no se notaría y "en gran medida" pasaría por un mecanismo reducible a la zoología (posición de Leroi-Gourhan).

A pesar de que Leroi-Gourhan parece haber considerado lo que implicaba esta ruptura (técnica) para el hombre, no logra profundizar en la discusión de este origen y restablece distinciones clásicas (entre Homo Faber y Homo Sapiens, entre lenguaje técnico y simbólico, anticipación técnica y reflexiva, entre concreto y abstracto) para justificar un segundo y "verdadero" origen del hombre. A partir de este momento, las capacidades que emergieron con el proceso de exteriorización que es la técnica (anticipación, lenguaje) se vuelven sólo "técnicas", "limitadas", “concretas" ("inauténticas" para hablar como Heidegger). Al mismo tiempo, todas estas capacidades se repiten ya no de manera limitada sino propiamente humana en la anticipación de la muerte (ser-para-la-muerte), el lenguaje simbólico y la reflexión abstracta que emergen con Homo Sapiens y el fin del desarrollo cortical. La pregunta que hace Stiegler es la siguiente: ¿si estas capacidades (técnicas, idiomáticas, cognitivas) estaban presentes en forma rudimental en el Zinjantropus y que su desarrollo culmina en Neantropus, 'colocar' el origen del hombre en Neantropus no será confundir el proceso con el resultado? ¿No será no querer admitir que el origen está en el proceso (y en la inauguración ubicua de este proceso) y no en el resultado que muestra de manera patente que el proceso ha cambiado? 
Para ilustrar esta crítica podemos hacer una analogía con la enfermedad de Alzheimer. El problema que ve Stiegler en la argumentación de Lerio-Gourhan es que si las capacidades cerebrales del Neantropus (técnicas, idiomáticas, cognitivas, simbólicas) estaban presentes en forma rudimental en el Zinjantropus, 'colocar' el origen del hombre en el Neantropus corresponde a confundir el proceso con el resultado. Sería como creer, en el caso de la enfermedad de Alzheimer, que la enfermedad empieza con el diagnóstico, aun cuando es un proceso que se ha venido desarrollándos mucho tiempo antes. Lo que se ve en el Neantropus, así como en el diagnóstico del Alzheimer, es una etapa de un proceso que se avista desde el Zinjantropo. Así, la propuesta de Stiegler es ver la novedad de lo humano más en el proceso de exteriorización técnica que en la substancia cerebral que resulta de este proceso, más en el cambio transductivo que implica esta exteriorización que en un comienzo simple o una causa única.

En suma, la tesis del autor es que la técnica y el hombre son co-originarios desde la 'primera' exteriorización técnica, y no dejan de serlo. Para sostenerlo, recurre al principio de transducción de Simondon y a la diferancia de Derrida.

\section{La individuación técnica y la individuación humana son co-originarias}

El origen relacional del hombre: exteriorización técnica e interiorización cognitiva

La tesis stiegleriana es que la diferancia del hombre, dentro de la diferancia de la vida, es la técnica. Con esto, podría preguntarse cuál es la relación especial entre la técnica y el hombre que hace que sean co-originarios y distintos del animal y de lo que podríamos llamar la tecnicidad de lo vivo. Frente a esto, hay que aclarar lo que Stiegler entiende por anticipación en lo humano: pues, dirá que el hombre es el único animal que hace técnica (exteriorización técnica) en la medida en que anticipa la muerte (interiorización cognitiva correlativa de la exteriorización técnica). Este saber de su muerte es tan originario como las prótesis técnicas que lo hacen humano y desde esta perspectiva cobra sentido el que el medio técnico es condición de posibilidad de la temporalidad humana (Cf Stiegler, 2003, p. 214).

Ahora bien, reiterando que Stiegler parece otorgarle una primacía constitutiva a la exteriorización técnica sobre la interiorización cognitiva humana (anticipación de 
la muerte), la pregunta que vuelve a plantearse es cómo entender este origen relacional entre la técnica y el hombre sin caer en un tecnocentrismo o en un antropocentrismo. En efecto, por un lado Stiegler asume que:

Si parece evidente que esta evolución ya no está simplemente determinada por la del quién en tanto que zoon, por el quién en tanto que ser vivo, el cual se diferencia como todos los demás seres vivos, entonces se deberá concluir que es más la evolución del qué lo que tiene a su vez un efecto retroactivo sobre el quién y dirige en cierta medida su propia diferenciación: el quién no se diferencia como todos los demás seres vivos, se diferencia a través de una diferenciación de lo no-vivo ( $\mathrm{y}$ un diferimiento de la muerte por esa diferenciación en la muerte), a través de una materia organizada aunque inorgánica, el qué. (Stiegler, 2003, p. 231)

Desde esta perspectiva, pareciera que la técnica (el qué) posee una primacía constitutiva sobre el hombre (el quién). Pero, por otro lado, Stiegler afirma, de acuerdo con la perspectiva transductiva simondoniana que "el quién inventa el qué tanto como aquel es inventado por éste” (Stiegler, 2003, p. 264).

Notamos una tensión entre dos interpretaciones ligeramente distintas de este origen relacional: una en la cual se enfatiza la primacía constitutiva del proceso de exteriorización técnica (sobre él de interiorización cognitiva) en la invención del hombre y otra en la cual estos dos procesos (interiorización y exteriorización) parecen desempeñar un papel equivalente, como si fueran dos procesos 'simétricos' o indistinguibles sino por el sentido que les damos a posteriori (después de la individuación en polos interior y exterior, por ejemplo).

Que Stiegler ponga el acento sobre el proceso de exteriorización más que sobre el de interiorización cognitiva (anticipación de la muerte) puede entenderse como un gesto que muestra el interés del autor por apartarse de la idea de un "origen sustancial" del hombre en su capacidad cerebral. Al final de todo, cuando Leroi-Gourhan asume el "verdadero origen" del hombre en el Neantropus, caería en esta aprehensión antropocéntrica que ve en el tamaño del cerebro la causa del comportamiento humano. Pero no por ello habría que caer en el otro extremo, es decir asumir que toda la constitución del ser humano recae sobre la técnica. Por consiguiente, la tarea interpretativa a la que nos vemos volcados sería la de precisar la simetría o asimetría entre estos dos 
procesos (exteriorización técnica e interiorización cognitiva) a través de los cuales Stiegler describe la ruptura provocada en la transducción vital por el advenimiento de la técnica y el hombre.

Respecto a la anticipación, que inicialmente asociaríamos al hombre, Stiegler precisa que:

Hay que distinguir aquí dos niveles de comprensión de la anticipación: la emergencia de esta posibilidad de anticipar, al nivel en el que la analizamos aquí, se opera en una casi-inmovilidad en relación a otro nivel que ya no será sólo ese tiempo de la anticipación, que podríamos denominar "operatorio", sino como un tiempo de anticipación tal que la misma forma de la anticipación se transforma ahí, se amplía ahí, y de una manera tal que el hombre deviene ahí, y no deviene lo que deviene la técnica. Habrá: -la anticipación sin la cual el hombre no podría fabricar ninguna herramienta; -la anticipación también en el sentido de que la fabricación de las herramientas no sólo se repite bajo la forma de un estereotipo, sino que evoluciona, se transforma, se diferencia. Pero, ¿se las puede separar?”. (Stiegler, 2003, p. 230)

Vemos que Stiegler distingue entre dos niveles de anticipación: del lado del hombre la anticipación corresponde a una temporalidad operatoria relacionada con las actividades humanas mientras que, del lado de la técnica, la anticipación corresponde a un marco para la anticipación humana, marco en el cual ésta evoluciona y se transforma. La evolución de este marco tecno-lógico para la anticipación humana a la vez depende de esta capacidad humana de anticipación, pero posee cierta autonomía respecto a esta escala (humana) de la anticipación. Desde esta perspectiva, pareciera que la anticipación tecno-lógica es el medio en el cual se desarrolla y madura la anticipación humana. Otra vez, estos dos procesos conectados transductivamente (el uno no existe sin el otro) parecieran asimétricos: las escalas operatorias de la anticipación humana y tecno-lógica no son las mismas y no están relacionadas de manera simétrica.

Esta tensión entre niveles de procesos diferentes, entre simetría y asimetría de los procesos de interiorización y exteriorización implicados en el origen transductivo del hombre y la técnica, la volvemos a encontrar en distintos momentos de la argumentación stiegleriana. Por lo tanto, aclarar lo que entendemos por simetría, asimetría y 
niveles de procesos en estas transducciones u orígenes relacionales, permitiría quizás resolver esta tensión que, en cierta medida, impregna todo el discurso de Stiegler.

\section{La diferencia humana dentro de la diferencia de la vida}

Para aclarar este punto, tenemos que regresar sobre la caracterización que realiza Stiegler del hombre y de la técnica a través de la diferancia y sobre la manera cómo, gracias a esta noción, pasa del animal al hombre. En este paso se juega la caracterización del origen relacional del hombre desde la animalidad.

La diferancia, desarrollada por Derrida para significar el proceso de la vida, será re-utilizada por Stiegler para significar la técnica como diferancia humana (que es la técnica) dentro de la diferancia (que es la vida). Esta manera de aprehender la vida, la técnica y el hombre no impone - aunque tampoco excluye- una jerarquía ontológica dentro de la vida y cuestiona la frontera entre animalidad (tecnicidad de lo vivo) y humanidad (técnica humana). Stiegler anticipa sobre el segundo tomo de La técnica y el tiempo (La desorientación, 2002) indicándonos que a esta relativización (animalidad - humanidad) corresponde también la relativización del privilegio acordado a la "escritura lineal alfabética" (Stiegler, 2003, p. 206) frente a otros modos de exteriorización (frente a otro tipo de huellas): la primacía de este tipo de escritura corresponde a una primacía de la palabra sobre la escritura y corresponde tanto al logo- como al fono-centrismo puestos en relieve y criticados en De la Gramatología (Derrida, 1978). Así el fono-logocentrismo corresponde a un antropomorfismo que le otorga un privilegio a la oralidad (lenguaje hablado) sobre la escritura, privilegio que corresponde también al dominio de la razón sobre la naturaleza y a la superioridad del hombre (de la palabra) sobre el animal (la huella).

La diferencia entre la escritura y la palabra es una separación fundamental entre el hombre y el animal, pues la escritura como una huella está al nivel del animal, mientras que el uso de la palabra sólo pertenece al hombre. Desde aquí, la escritura es una huella en exterioridad, es un proceso de individuación técnica, y la oposición entre hombre y animal corresponde a la oposición de la palabra y la escritura, correlativa a la oposición entre logos y téchne. Está es la oposición clásica. Pero, desde un paradigma distinto, nos interesa pensar en que no hay ruptura entre hombre y animal fundamentada en la oposición de téchne y episteme. Esto en el fondo implica relativizar la oposición entre el hombre y la técnica. 
Para Derrida la huella se sitúa a nivel de la vida y no es exclusiva del ser humano. El grama sería la estructura elemental de la huella como unidad significante para la vida. La historia del grama, que es la historia de la vida, corresponde a "un proceso de liberación de la memoria" en el cual se "ensancha la diferancia y la posibilidad de puesta en reserva" (Stiegler, 2003, p. 207). Así, para entender la emergencia del ser humano dentro de la vida, hay que estudiar la ruptura introducida por la técnica en la configuración del grama como diferancia. Para Stiegler, esta ruptura será vista e interpretada como una doble ruptura, correspondiendo a los "momentos" del Zinjantropus y del Neantropus en el proceso de hominización: es sobre esta doble ruptura y su interpretación - clásica - como doble origen que se centrará toda la lectura de Stiegler de Leroi-Gourhan y la mitigación de su análisis. Entre estos dos “personajes”, lo que parece jugarse es la cuestión del origen del hombre. Mientras que en el Zinjantropus se observa una co-evolución técnico-cerebral que sigue un ritmo genético lento, a partir del Neantropus la evolución técnica se acelera y se desacopla de la evolución cerebral, que se “estabiliza”.

La cuestión del paso de una co-evolución técnica-cerebro que se hace a un ritmo genético a una evolución tecno-lógica mucho más rápida y desacoplada de la evolución genética cerebral, es lo que ocupa la discusión con Leroi-Gourhan. ¿Cómo interpretar esta co-evolución y el desacoplamiento subsiguiente? ¿A partir de cuándo podemos hablar de la invención o del nacimiento del hombre?

\section{Simetría y asimetría en el origen relacional y la evolución técnica-hombre}

\section{Vida, diferancia y exteriorización técnica}

Stiegler aborda inicialmente la cuestión del origen del hombre siguiendo a la perspectiva zoológica evolutiva introducida por Leroi-Gourhan, lo que le permite abordar esta pregunta "fuera de todo antropologismo" (Stiegler, 2002, p. 205), es decir, fuera de toda pre-concepción acerca del hombre. Lo clave, en este acercamiento zoológico a la pregunta por el origen del hombre, es preguntarse por el proceso de fabricación (invención) del hombre desde la vida - y en especial, desde la animalidad (zoo). La cuestión del hombre tiene que ver con la especificidad de su fabricación en el seno de la evolución de la vida. Por supuesto, se asume aquí un punto de vista evolutivo desde el cual pensamos el origen del hombre como el inicio de una 'ramificación' en 
el 'árbol de la vida terrestre'. Desde este punto de vista, y en primera aproximación, aclarar la cuestión del origen del hombre conduciría a tratar de contestar dos tipos de preguntas: 1) ¿qué es la vida? Y 2) ¿Cuál es la especificidad del hombre dentro de la vida? Sin embargo, adoptar una vía zoológica para aclarar el origen del hombre, es ya asumir implícita o explícitamente cierta manera de contestar la primera pregunta planteada, sobre el sentido de la vida. ¿Hasta qué punto el discurso de Stiegler explicita la concepción de la vida sobre la cual se apoya para pensar la especificidad humana? ¿En qué medida (y cómo) esta concepción de la vida se relaciona con la especificidad humana vista desde el fenómeno de la exteriorización técnica (tesis que defiende Stiegler)? Esta discusión se centrará sobre estas dos preguntas.

Respecto a la primera pregunta, y además de Leroi-Gourhan, Stiegler apela a dos fuentes filosóficas para explicitar y asentar la concepción de la vida que adopta. En La técnica y el tiempo (tomo 1, El pecado de Epimeteo) y particularmente en la parte que estamos discutiendo (tercer capítulo de la primera parte) Stiegler se apoya explícitamente en la diferancia derridiana. En otras partes de La técnica y el tiempo o en otro texto relacionado (Stiegler, 1998), Stiegler se apoya sobre la individuación de Simondon para pensar la vida. Nos centraremos aquí sobre la discusión del uso que hace Stiegler de la diferancia derridiana tanto para abordar la vida (pregunta 1) como para abordar la especificidad humana dentro de la vida (pregunta 2).

En La técnica y el tiempo (primera parte, capítulo 3, 1- La diferancia* del hombre), Stiegler se detiene sobre una cita de Derrida que va a discutir y desde la cual afianzará su concepción de la vida y del hombre desde la diferancia. En esta cita, modificada por Stiegler (uso de corchetes), Derrida sostiene que "la huella es la diferancia que abre el aparecer y la significación [que articula] lo vivo sobre lo no vivo en general, [que está en el] origen de cualquier repetición"” (Stiegler citando a Derrida, 2002, p. 210). Enseguida, Stiegler considera que:

Existe una especie de indecisión sobre la diferancia: es la historia de la vida en general, pero (sólo) se da como (datando de) después de la ruptura, incluso cuando esta ruptura sea, si no nada, al menos mucho menos de lo que significa la división clásica entre humanidad y animalidad”. (Stiegler, 2002, p. 210)

La ruptura en cuestión corresponde al inicio del proceso de exteriorización técnica que Leroi-Gourhan asocia al Zinjantropus y que Stiegler entiende como el punto a 
partir del cual empieza a surgir o inventarse el hombre. Así Stiegler considera que en la articulación "de lo vivo sobre lo no vivo", lo no vivo corresponde a la huella (resultado de una exteriorización de lo vivo en lo no vivo) que es también, desde su perspectiva, la técnica. Por lo tanto, la huella, entendida como exteriorización técnica, surgiría con el hombre. Pero, por otro lado, Derrida considera que la huella se ubica al nivel de la vida y que no es específica del ser humano. La indecisión que percibe Stiegler en la cita de Derrida se podría por lo tanto reformular de la siguiente manera: si la diferancia corresponde a la exteriorización técnica y que ésta es específica del hombre, entonces no podría en principio servir para describir la vida. Por lo tanto, desde esta perspectiva uno se preguntaría: ¿la diferancia es específica de la vida o del hombre?, ¿La huella, como "articulación de lo vivo sobre lo no vivo" es lo mismo que la exteriorización técnica entendida como “organización de la materia inorgánica”?

Las cosas no son tan sencillas y tenemos que adentrarnos en la manera cómo Stiegler trabaja esta indecisión para fundamentar su tesis de la invención del hombre a partir de la técnica. Al recordar que "la physis como vida ya era diferancia" (Stiegler, 2002, p. 211), considera que podemos entender la técnica como diferancia humana dentro de la diferancia (que es la vida). Esta 'nueva diferancia' correspondería a la exteriorización técnica, como 'diferancia de diferancia' o exteriorización de lo vivo en lo no vivo. Lo 'nuevo' de esta diferancia entraña aquí un doble sentido: es a la vez repetición (de la diferancia de la vida) y novedad (respecto a la diferancia de la vida). Esta visión recursiva (en el sentido matemático de la recursión: 'diferancia de diferancia') sobre la vida y el hombre conduce Stiegler a precisar la especificidad humana dentro de la vida de la manera siguiente:

"el hombre se inventa en la técnica inventando la herramienta - exteriorizándose tecnológicamente. Ahora bien, el hombre es aqui el "interior": no hay exteriorización que no designe un movimiento del interior hacia el exterior. Sin embargo, el interior es inventado por ese movimiento: no puede, por lo tanto, precederlo. Por consiguiente, interior y exterior se constituyen en un movimiento que inventa al uno y al otro a la vez: un movimiento en el que se inventan el uno en el otro, como si hubiera una mayéutica tecno-lógica de eso que se llama el hombre. El interior y el exterior son lo mismo; el dentro es el fuera puesto que el hombre (el interior) es definido esencialmente por la herramienta (el exterior). Sin embargo esta doble constitución es también la de una oposición entre interior y exterior -o produce una ilusión de sucesión" (Stiegler, 2002, pp. 213-214). 
Enseguida, Stiegler aclara que la racionalidad operando en esta "mayéutica tecnológica del hombre" se desarrollará como tal en la segunda parte (del primer tomo) como "olvido originario, de la epimeteia como retraso, del pecado de Epimeteo" (Stiegler, 2002, p. 214). Ahora bien, queremos tratar de delimitar lo que nos parecen unos avances sustanciales en cuanto a la comprensión de la vida, la técnica y el hombre y lo que, a nuestro parecer sigue haciendo pregunta o preguntas nuevas que surgen sobre la vida, la técnica y el hombre desde esta perspectiva.

\section{Diferancia de diferancia: novedad y repetición}

El primer punto que queremos resaltar es que Stiegler no se detiene sobre 'la diferancia de la vida' como para precisar su sentido respecto a la 'diferancia (exteriorización) técnica' dado que re-incorpora la descripción que Derrida propone de la primera en la segunda (como diferancia de diferancia). Así, uno se preguntaría en qué medida la diferancia técnica entendida como "exteriorización de lo vivo en lo no vivo" y a la vez como "doble constitución” del hombre (interioridad) y de la técnica (exterioridad) no corresponde también a una determinación de lo vivo. Stiegler parece asumir que la técnica entendida como este proceso de exteriorización es la novedad de la diferancia del hombre dentro de la diferancia de la vida. Pero hemos ya indicado que en esta visión recursiva, esta novedad es también repetición. Por lo tanto, nos preguntamos hasta qué punto lo que Stiegler presenta como La novedad de la diferancia técnica no puede verse también como repetición, por la técnica y en lo humano, de un movimiento de exteriorización ya presente de alguna manera en la vida.

Por su lado, Derrida concebía la vida misma como diferancia, exteriorización en la huella, sin interioridad previa. Stiegler desplaza y específica esta propuesta a nivel del hombre. ¿En qué medida este desplazamiento y esta especificación de la diferancia conducen efectivamente a precisar la diferancia humana (la técnica) dentro de la vida? ¿En qué medida no oculta, a la vez, la pregunta por la diferancia de la vida sobre la cual se asienta esta nueva diferancia? Así, uno podría preguntar si lo que Stiegler considera acerca de la técnica humana como movimiento de exteriorización por el cual se co-originan, co-constituyen y co-evolucionan la técnica (exterioridad) y el hombre (interioridad) no existe de alguna manera a nivel de la vida, en lo biológico.

Si consideramos distintos autores que intentan abordar la vida desde una perspectiva relacional como la que propone Stiegler después de Derrida, podemos hacer una 
observación que 'ilumine' un camino para dar cuenta del énfasis puesto por Stiegler sobre la exteriorización. Estos autores son Gilbert Simondon con La individuación ${ }^{1}$, Hans Jonas con El fenómeno de la vida (1966) y Francisco Varela con la teoría biológica enactiva. Sin entrar en los detalles de las propuestas de estos tres autores para pensar la vida, queremos indicar que, cada uno a su manera, desarrolla una perspectiva relacional ("transducción" en Simondon, "enacción" en Varela, "libertad en la necesidad" para Jonas) sobre el ser de la vida que rompe con la visión sustancialista (la que todavía prevalece cuando hablamos, por ejemplo de 'materia orgánica e inorgánica' para hablar de 'materia viva y muerta'). Esta perspectiva relacional les conduce a pensar la vida como auto-constitución de una interioridad viva ontológicamente distinta de la materia que la constituye en cada momento. No es nuestro objetivo discutir aquí los detalles y las diferencias entre estas propuestas sino que queremos resaltar que estos autores, para dar cuenta de la vida, ponen el acento sobre la constitución de una interioridad viva distinta del exterior material mientras que Stiegler pone el acento, para dar cuenta del origen del hombre, sobre la constitución de una exterioridad técnica distinta de la vida.

Así, mientras que en el caso del hombre la exteriorización técnica pareciera ser la que modula la interiorización cognitiva, en el caso de la vida pareciera ser la interioridad viva la que modula (regula) la relación con la exterioridad material. Mejor dicho, en los dos casos pareciera que estamos tratando con un ser relacional pero que no funciona de la misma manera, en el mismo sentido o a la misma escala. Para seguir en este sentido, tenemos que insistir sobre la perspectiva ontológica relacional adoptada por estos autores: los polos en relación (la técnica y el hombre, la interioridad viva y la exterioridad material) no preceden a su relación sino que se co-constituyen en y por la relación. Desde esta perspectiva relacional, no aparece inicialmente una polarización sobre un movimiento (interiorización o exteriorización) o la constitución de un polo (interioridad o exterioridad) en particular. Por lo tanto, ¿de dónde proviene esta asimetría entre exteriorización (técnica) e interiorización (cognitiva) en Stiegler y entre interioridad (viva) y exterioridad (material) en los otros autores mencionados? Si cómo lo hemos dicho, estos autores asumen una perspectiva ontológica relacional, ¿por qué ponen el acento más sobre un polo (interioridad o exterioridad) o un proceso (interiorización o exteriorización) que sobre el otro?

1 Sobre el cual se apoya ampliamente Stiegler por otro lado: ver Tiempo e individuaciones técnica, psíquica y colectiva en la obra de Simondon (Stiegler, 2012). 
La hipótesis que queremos presentar y desarrollar para tratar de contestar esta pregunta es la siguiente: en el caso de la vida, la interioridad viva resulta de la interiorización, en la estructura y el funcionamiento del ser vivo, de regularidades relacionales con el medio exterior; mientras que en el caso del hombre, lo determinante radicaría más bien en la exteriorización de regularidades relacionales con el medio exterior en la estructura y el funcionamiento del objeto técnico. Si ahora tratamos de integrar estas dos consideraciones podríamos pensar que: 1) lo que se interioriza en la organización del ser vivo son regularidades relacionales con el medio exterior que lo configuran en su interioridad y funcionamiento como ser vivo y 2) lo que se exterioriza en la organización del objeto técnico son regularidades relaciones de 'orden superior', que se configuran en la relación entre la interioridad viva y el mundo exterior. La técnica, aunque inorgánica, no viva, estaría sin embargo pro-movida e in-formada por el régimen relacional correspondiente a la vida. Así podríamos entender que, como organización de la materia inorgánica, el objeto técnico no proviene de una interioridad humana que le pre-existe sino de la exteriorización del funcionamiento relacional de lo vivo en lo material. Quizás podríamos decir que la vida, desde esta aprehensión relacional, pre-existe a la técnica en la medida en que ésta nace como exteriorización de aquella. Esta exteriorización crearía nuevas condiciones de individuación de lo vivo, a partir de un medio pre-individual pre-in-formado por él, que llamamos 'la técnica y el hombre'. Desde esta perspectiva, tendría sentido hablar de la diferancia técnica del hombre en el doble sentido de 'novedad y repetición' respecto a la diferancia de la vida.

\section{Tecnicidad de lo vivo y técnica humana como formas reguladoras del ser vivo y del ser humano}

Ezequiel Di Paolo, que es un investigador de primer plano trabajando en el desarrollo de la teoría enactiva planteada por Varela y Maturana, afirma en El enactivismo y la naturalización de la mente (Di Paolo, 2013) que "cuando los mecanismos adaptivos operan más allá de la frontera física del organismo, (...) terminan regulando su acople con el entorno" y que en este caso:

Nos desplazamos conceptualmente desde una perspectiva de acople estructural (que es esencialmente simétrica e implica que tanto sistema como entorno se influyen mutuamente sin pérdida de viabilidad) hacia una perspectiva de comportamiento (concepto asimétrico según el cual el organismo es el origen de una regulación en los parámetros y condiciones del acople estructural). Esta 
regulación de las interacciones con el entorno dentro del contexto de búsqueda de sentido es lo que nos permite definir a ciertos sistemas autopoiéticos y adaptivos como agentes (Barandiaran, Di Paolo, Rohde, 2009). El concepto de agencialidad es asimétrico porque implica una regulación del acople, el cual ya de por sí es de entrada un bucle de mutuas influencias entre organismo y entorno, no su mera existencia. La modulación implica un segundo bucle de orden superior siguiendo las normas dadas por la adaptividad del agente (Figura 1). (Di Paolo, 2013, p. 15)

Figura No. 1 Funciones de Agencialidad

A

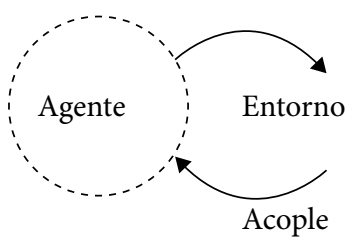

B

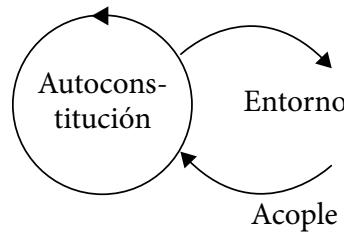

C

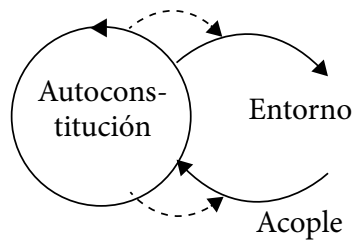

A) Un agente definido por convención en interacción con su entorno, situación tradicional en el funcionalismo. B) Un sistema auto-definido a través de un proceso de auto-mantenimiento (como la autopoiesis). C) Definición de un agente: un sistema auto-definido que regula de manera adaptiva su acople con el entorno (Di Paolo, 2013, p. 16).

Citamos este extracto de la propuesta enactiva de Di Paolo porque, a nuestro parecer, converge hacia lo que propusimos para tratar de entender la vida desde una perspectiva relacional. En efecto, planteamos que la diferancia de la vida radica en la interiorización de regularidades relacionales con el medio en la estructura y el funcionamiento del ser vivo. Cuando hablamos de "regularidades relacionales", nos referimos a un concepto "simétrico" como él de "acople estructural", que no implica una polarización sobre alguno de los polos en relación o elementos acoplados. Sin embargo, cuando hablamos de “interiorización” - de estas regularidades relaciones - eso significaría que existe una ruptura en la simetría considerada y que lo relacional posee ahora una orientación; de la misma manera, hablar de "regulación" del acople estructural implica una ruptura de simetría en la noción de acoplamiento entre el sistema vivo y el entorno. Por lo tanto, lo diferante de la vida radicaría en esta ruptura de simetría, en esta orientación de su constitución relacional (interiorización) y de su funcionamiento acoplado (regulación desde el interior). 
Ahora bien, lo que Di Paolo define como "agencialidad de un sistema vivo" corresponde precisamente a esta asimetría, a la regulación, desde el interior, de su acople estructural con el entorno. Por otro lado, hemos visto que en el caso del ser humano y la técnica, Stiegler planteaba que es la individuación técnica la que regula a la individuación humana (y no al contrario). Eso es claro en Tiempo e individuación en la obra de Simondon (Stiegler, 2012) donde Stiegler le critica la falta de articulación entre individuación psíquica y colectiva (humana) e individuación técnica:

\begin{abstract}
Simondon no articuló estos dos aspectos de su trabajo. Sin embargo, ¿no articula la técnica, como huella y herencia, la individualidad psíquica y colectiva en lo que denominaremos aquí "epifilogénesis"? A partir de Heidegger y Derrida, se trata de designar el irreductible avance de la técnica que, diferencia temporal originaria, forma el horizonte de cualquier individuación psicosocial. (Stiegler, 2012, pp. 133-134)
\end{abstract}

Según Stiegler, la técnica, en cuanto "memoria epifilogenética" constituye el medio preindividual desde el cual se individua psíquica y colectivamente el ser humano (Stiegler, 2012, p. 140). Desde esta perspectiva, sería la exteriorización técnica la que regularía a la individuación humana, y no lo contrario como se suele pensar desde la tradición filosófica. Siguiendo a Stiegler, en la individuación humana el papel regulativo, la regulación, estaría del lado de la exterioridad tecno-lógica mientras que en el caso de la vida hemos visto que éste aparecía como estando del lado de la interioridad viva y de lo que Di Paolo define como la “agencialidad de un sistema vivo”. ¿Será posible pensar que lo que inaugura el paso de la vida al hombre radica en este cambio de régimen relacional, en esta ruptura de simetría diferante por la cual opera un nuevo régimen regulativo que no está orientado del interior hacia el exterior sino del exterior (tecno-lógico) hacia el interior (humano)? A la "agencialidad interior" del ser vivo correspondería la "tecnicidad exterior" del ser humano como exteriorización de este nivel vital. Pero si la "agencialidad interior" corresponde a un "bucle de orden superior" por el cual se entiende, más allá de la noción simétrica ( $\mathrm{y}$ funcionalista) de acople estructural, al ser vivo como diferante de su entorno material, dotado de un comportamiento propio, la "tecnicidad exterior" del ser humano también debería ser vista como un "bucle de orden superior" por el cual se entiende al hombre como diferante de la vida.

Otra pregunta que surge de esta comprensión, es si no podríamos también llamar a la "agencialidad de un sistema vivo", tecnicidad de lo vivo. En efecto, desde una 
perspectiva formal, lo que permite distinguir entre agencialidad de un sistema vivo y técnica humana es la diferencia en la orientación de la regulación que opera cada una de estas nociones: la agencialidad está orientada del interior (vivo) hacia el exterior (material) mientras que la técnica estaría orientada del exterior (tecno-lógico) hacia el interior (humano). Pero si lo que se exterioriza en la técnica es la agencialidad de la vida, la técnica vendría a sacar su potencial relacional exterior de una propiedad interior de la vida que podríamos entonces llamar “tecnicidad de la vida”. La técnica humana vendría a repetir la tecnicidad interior de la vida de nuevo, en exterioridad, dando lugar a una nueva forma relacional de vida que llamamos el hombre.

Por su lado, cuando Stiegler compara el devenir del objeto técnico con él del ser vivo, considera que: "La concretización del objeto técnico es su devenir-individuo, es decir, su organización como devenir-indivisible. Se trata de una dinámica casi biológica: mientras que el ser vivo mantiene su unidad, el objeto técnico tiende a alcanzarla" (Stiegler, 2012, p. 136).

Si lo que le permite al ser vivo "mantener su unidad" es lo que Di Paolo llama agencialidad, en qué medida no podríamos decir que el objeto técnico tiende a la agencialidad. A la vez, eso significa que nunca la alcanza del todo y que a diferencia del ser vivo, que por la agencialidad es autónomo, el objeto técnico nunca podrá superar su heteronomía constitutiva. Esta heteronomía irreducible y constitutiva significa que el objeto técnico requiere del ser humano como ser vivo autónomo que puede regular su acople con el entorno como para funcionar con esta agencialidad en exterioridad que Stiegler llama memoria epifilogenética. La tecnicidad de la vida sería esta agencialidad autónoma del ser vivo mientras que la técnica humana radicaría en la exteriorización de esta agencialidad, que vendría así a configurarse como un medio pre-individual permitiendo una nueva auto-nomía que sería la individuación humana. La tecnicidad de lo vivo (auto-noma) y la técnica humana (hetero-noma) vendrían a ser condiciones necesarias para entender la mayéutica tecno-lógica del ser humano.

\section{Conclusión}

Desde esta perspectiva podemos reconsiderar la cuestión del doble origen del hombre que Stiegler le critica a Leroi-Gourhan. Eso es la cuestión de lo que pasa en el paso del Zinjántropus al Neántropus. Con el inició de la exteriorización de la tecnicidad de lo vivo en objetos técnicos, el hombre surge como esta nueva autonomía del 
sistema vivo pero sigue heredero, desde el plano evolutivo, de su estructura biológica comportamental, de la tecnicidad de lo vivo. Luego, a medida que esta estructura biológica evoluciona por su acople estructural con un medio devenido técnico, su comportamiento se ve cada vez más moldeado por y adaptado a este medio tecnológico. Para usar una metáfora artística es como si, con la primera herramienta (con Zinjántropus), el ser vivo empezaba a esculpirse como hombre y que, después de un tiempo (con Neántropus), ya hubiera acabado su obra. En este sentido, cuando la evolución biológica alcanza sus límites (con Neántropus), es que el carácter bio-lógico del hombre ya se confunde completamente con su carácter tecno-lógico, es decir que el ser humano radica en su acople estructural biológico con un medio exterior tecno-lógico. Con esto, sería plausible pensar que esta nueva forma de acople también corresponde a una nueva escala de en-gramación (memoria) y de funcionamiento biológico en el ser humano. La epigenética, que corresponde en biología al estudio de los mecanismos de regulación de la expresión genética, podría ser pensada como el registro biológico interior en el cual se marca esta orientación del acople estructural del hombre con el medio tecno-lógico. Eso supondría que el carácter tecno-lógico exterior del ser humano se vería reflejado en su funcionamiento epigenético interior. Dejamos en suspenso esta discusión porque requeriría, para ser prolongada y precisada, introducir explicaciones y conceptos biológicos en la visión tecno-lógica (epifilogenética) propuesta por Stiegler.

Por fin, ¿cuál podría ser la pertinencia de semejante acercamiento a la vida, la técnica y el hombre para la fundamentación de una bioética global capaz de regular el desarrollo tecno-lógico de la humanidad en pro de la vida? Quizás la respuesta a esta pregunta radicaría, desde la perspectiva desarrollada en este escrito, en la manera de pensar relacionalmente el carácter regulativo de la mayéutica tecno-lógica del ser humano. Eso sería un poco equivalente a pensar el problema de la gobernabilidad (regulación) de manera des-centralizada o relacional, es decir sin partir de un polo o centro regulador constituido antes del sistema que se pretende auto-gobernar - y aunque eso puede conducir a la formación de centros y polos efectivamente reguladores del sistema en cuestión. 


\section{Referencias}

Di Paolo, E. (2013). El enactivismo y la naturalización de la mente. En: D. P. Chico and M. G. Bedia (2013), Nueva ciencia cognitiva: Hacia una teoría integral de la mente. Madrid: Plaza y Valdes Editores.

Simondon, G. (1989). L'individuation psychique et collective. Paris, Aubier Montaigne.

Simondon, G. (1969). Du mode d'existence des objets techniques. Paris: Aubier Montaigne.

Simondon, G. (1964). L'individu et sa genèse physico-biologique. Paris: PUF, col. Epiméthée.

Stiegler, B. (1994). La technique et le temps - tome 1 : La faute d'Epiméthée. Paris: Galilée.

Stiegler, B. (1996). La technique et le temps - tome 2 : La désorientation. Paris: Galilée.

Stiegler, B. (1998). Temps et individuation psychique et collective dans l'ouvre de Simondon.

Stiegler, B. (2001). La technique et le temps - tome 3 : Le cinéma et la question du malêtre. Paris: Galilée.

Stiegler, B. (2002). La técnica y el tiempo, tomo I: El pecado de Epimeteo. Traducción de Beatriz Morales Bastos. Hondarribia: Ediciones Hiru.

Stiegler, B. (2012). Tiempo e individuaciones técnica, psíquica y colectiva en la obra de Simondon. TRILOGÍA (6), pp. 133 - 146. Traducción de Rodrigo Zapata Cano desde el francés: Temps et individuation technique, psychique et collective dans l'œuvre de Simondon. Intellectica (1998), Vol.1/2, (26-27), pp. 241-256. 